\title{
Strong-coupling theory of two dimensional large bipolarons in elliptical quantum dots
}

\author{
R.T. Senger ${ }^{\mathrm{a}}$ and A. Erçelebi \\ Department of Physics, Bilkent University, 06533 Ankara, Turkey
}

Received 30 July 2001 and Received in final form 28 January 2002

\begin{abstract}
In the limit of strong electron-phonon coupling, we analyze the stability of two dimensional bipolarons in a two-axis elliptic potential well of harmonic boundaries. The confined two-polaron wavefunction adopted here makes the electrons to form either a bipolaronic bound state or go into a composite state of two separated polarons bounded inside the same potential well. The methodology involves the mean polaron-polaron separation treated as an adjustable parameter to be determined variationally. By tuning the barrier slopes of the confining potential we obtain an explicit tracking of the criterion for bipolaron stability encompassing the particular cases of a two dimensional circular dot or a planar strip-like quantum well wire. We observe that, while an increased degree of confinement enhances bipolaronic stability, the effect of anisotropy is to inhibit bipolaron formation.
\end{abstract}

PACS. 71.38.Fp Large or Fröhlich polarons - 71.38.Mx Bipolarons

\section{Introduction}

In selective materials, depending on the relevant dielectric properties, two electrons interacting with the medium and with one another through exchange of virtual phonons can lead to the formation of a stable bound state termed a bipolaron. The first qualitative discussion on the formation of two-electron bound states in a crystal was given by Pekar [1]. The quantitative description of the problem was later provided by Vinetskii and Gitterman [2]. An enormous amount of the preceding literature [3-28] published within the context of Fröhlich large bipolarons has led to the evidence that bipolaronic bound states of two electrons can indeed take place under critical circumstances characterized by the Coulomb repulsion and the electron-phonon coupling strengths. Described qualitatively, one has simultaneously the Coulomb aspect and the phonon-coupling counterpart of the problem to act in opposition against one another and in an interrelated manner so as to modify strongly the overall nature of the effective interaction between the electrons and lay out a totally distinguishing characterization of the electron pair compared with the case of bare electrons. Adopting different models and approximating theories, several aspects of the polaronbipolaron transition have been studied rather extensively as functions of the material dielectric properties in three, two [16-20], and even lower dimensionalities pertaining to quantum wires and dots [21-28].

The bipolaron concept has a considerable appeal and the interest in this subject continues unabatedly pertain-

\footnotetext{
a e-mail: senger@physics.emory.edu
}

ing to the investigation of a possible bipolaronic mechanism for high $-T_{c}$ superconductivity [29] and in the context of Bose-Einstein condensation of large bipolarons into a superfluid [30]. In the present work we refrain ourselves from discussing any pertinence to the many particle aspects of the bipolaron gas and limit our attentions only to the one bipolaron case with most emphasis devoted to the formal theoretical counterpart of a model calculation applied to two dimensional bipolarons in elliptical dots. For a brief discourse on (bi)polarons the reader is referred to the review articles by Devreese [31] and by Alexandrov and Mott [32].

In this paper we would like to provide a variational scheme to determine the critical conditions under which a bipolaronic state of two electrons can be realised. In our treatment of the Fröhlich interaction we choose to use the strong coupling polaron approximation, since the formation of bipolarons requires large values of the electronphonon coupling constant so as to sustain the phononmediated binding and withstand against the strong repulsive Coulomb interaction. Fortunately, the adiabatic theory we follow is motivated by the recent advances in producing nanocrystals with strong ionic coupling [33-35]. Moreover, the pseudo-enhancement of the electron phonon coupling in confined media is an additional complement supporting the applicability of the strong coupling theory. The key feature of the present formalism will be to set up a variational coherent phonon state for each electron as centered at the mirror image positions on either side of the origin, located respectively at $\pm \frac{1}{2} \boldsymbol{r}_{0}$. Thus, introducing $\left|\boldsymbol{r}_{0}\right|$ to represent the distance between the polaron centers, the procedure will rely on the determination of 
the optimal relative position coordinates which we intend to determine variationally in the foregoing calculations. Evidently, the case in which $\boldsymbol{r}_{0}$ comes out zero should refer to the bipolaron state where the charge density profiles of the electrons map on one another positioned centrally at the origin and clothed entirely in common by the lattice polarisation field.

The underlying motivation which makes us tempted to impose the relative polaron-polaron distance to take part in the variational model as an additional parameter originates from our attempts to study the bipolaron problem in the dot type confinement. In the peculiar case of an electron pair in a quantum dot the alternative of the bipolaron state is the case where the phonon-mediated binding breaks up and the electrons are repelled apart in opposite directions from their common center of mass, yet remain in a finite optimal separation yielding a description consisting of two individual but nearby polarons bounded within the dimensions allowed by the barriers of the dot potential. Clearly, the extreme variational limit $r_{0} \gg 1$ should only be realized in higher dimensional configurations such for instance, as in quantum wires or in the entire two dimensional space, where the electrons are totally free along at least one spatial direction.

We shall devote our attention to the study of formation of bipolarons in strict two dimensions confined within a deformable two-axis potential well of parabolic boundary strengths, given in usual polaron units $\left(m^{\star}=\hbar=\right.$ $\left.\omega_{\mathrm{LO}}=1\right)$ by

$$
V_{\mathrm{conf}}(x, y)=\frac{1}{2}\left(\Omega_{x}^{2} x^{2}+\Omega_{y}^{2} y^{2}\right)
$$

in which the dimensionless frequencies $\Omega_{x}$ and $\Omega_{y}$ serve for the measures of the degree of confinement of the electrons along the respective $x$ and $y$ axes. Clearly, by tuning these frequencies one attains a trace of the variational upper estimate for the energy of the two polaron complex and the corresponding criterion for bipolaronic stability interpolating between the entire space of two dimensions and the particular geometric configurations of a circular planar dot and a thin strip-like planar quantum well wire. In the following we shall take $\Omega_{x} \leq \Omega_{y}$, and thus, conveniently set $\boldsymbol{r}_{0}=x_{0} \hat{\mathbf{x}}$.

\section{Theory}

The Hamiltonian describing the confined two dimensional electron pair coupled to LO-phonons is given by

$$
H=H_{e}+\sum_{q} a_{q}^{\dagger} a_{q}+\sum_{j=1,2} \sum_{q} V_{q}\left(a_{q} \mathrm{e}^{\mathrm{i} \boldsymbol{q} \cdot \boldsymbol{r}_{j}}+a_{q}^{\dagger} \mathrm{e}^{-\mathrm{i} \boldsymbol{q} \cdot \boldsymbol{r}_{j}}\right)
$$

where

$$
H_{e}=\frac{1}{2} \sum_{j=1,2}\left(p_{j}^{2}+\Omega_{x}^{2} x_{j}^{2}+\Omega_{y}^{2} y_{j}^{2}\right)+\frac{U}{\left|\boldsymbol{r}_{1}-\boldsymbol{r}_{2}\right|} .
$$

In the above, $a_{q}^{\dagger}\left(a_{q}\right)$ is the phonon creation (annihilation) operator and $\boldsymbol{r}_{j}=x_{j} \hat{\mathbf{x}}+y_{j} \hat{\mathbf{y}} \quad(j=1,2)$, are the positions of the electrons. Similarly, $\boldsymbol{p}_{j}(j=1,2)$, denote the respective two dimensional momenta. The interaction amplitude is related to the phonon wavevector $\boldsymbol{q}$ through $V_{q}=(\sqrt{2} \pi \alpha / q)^{1 / 2}$. The dimensionless constants of the Coulomb interaction $U$ and of the electron-phonon coupling $\alpha$ are related by the equation

$$
U=\frac{e^{2}}{\epsilon_{\infty}}=\frac{\alpha \sqrt{2}}{1-\eta}
$$

in which parameter $\eta$ is the ratio of the high frequency and static dielectric constants of the material, given by

$$
\eta=\epsilon_{\infty} / \epsilon_{0}<1 \text {. }
$$

In the variational approximation that we follow here, we use the conventional trial ansatz of the adiabatic polaron theory where we write the total wavefunction to consist of a part relevant to the phonon variables and a part which contains the particle coordinates only, i.e.,

$$
\Psi\left(x_{0}\right)=\Phi\left(\boldsymbol{r}_{1}, \boldsymbol{r}_{2} ; x_{0}\right)\left[\prod_{j=1,2} S_{j}\left(x_{0}\right)\right]|0\rangle
$$

where $|0\rangle$ denotes the phonon vacuum state, and

$$
\left\{\begin{array}{l}
S_{1}\left(x_{0}\right) \\
S_{2}\left(x_{0}\right)
\end{array}\right\}=\exp \sum_{q} V_{q} s(q, \Phi)\left\{a_{q} \mathrm{e}^{ \pm \mathrm{i} \frac{1}{2} q_{x} x_{0}}-a_{q}^{\dagger} \mathrm{e}^{\mp \mathrm{i} \frac{1}{2} q_{x} x_{0}}\right\}
$$

are the displaced oscillator operators intended to yield the most efficient polarization fields for either electron, centered at $\pm \frac{1}{2} x_{0} \hat{\mathbf{x}}$.

Compatible with the lattice part of the trial wavefunction (6) we construct the particle part $\Phi\left(\boldsymbol{r}_{1}, \boldsymbol{r}_{2} ; x_{0}\right)$ in a form consisting of two "shifted" one-electron Gaussians along the $x$ axis peaked correspondingly at $\pm \frac{1}{2} x_{0}$, i.e.,

$$
\Phi\left(\boldsymbol{r}_{1}, \boldsymbol{r}_{2} ; x_{0}\right)=g\left(\left|\boldsymbol{r}_{1}-\boldsymbol{r}_{2}\right|\right) G\left(\boldsymbol{r}_{1}-\frac{1}{2} x_{0} \hat{\mathbf{x}}\right) G\left(\boldsymbol{r}_{2}+\frac{1}{2} x_{0} \hat{\mathbf{x}}\right)
$$

where $g\left(\left|\boldsymbol{r}_{1}-\boldsymbol{r}_{2}\right|\right)$ is the Coulomb correlation function of Jastrow type.

An optimization of

$$
E_{g}=\left\langle\Psi\left(x_{0}\right)|H| \Psi\left(x_{0}\right)\right\rangle
$$

with respect to $s(q, \Phi), x_{0}$ and the further variational parameters contained in $\Phi\left(\boldsymbol{r}_{1}, \boldsymbol{r}_{2} ; x_{0}\right)$ corresponds to the adiabatic self-trapping description of the polarons where the charge density fluctuations of the electrons and the induced polarization field influence mutually each other in such a way that a stable relaxed state of the "twoelectron + phonon" complex is eventually attained.

Transforming to a representation in the center of mass and relative coordinates:

$$
\boldsymbol{R}=\frac{1}{2}\left(\boldsymbol{r}_{1}+\boldsymbol{r}_{2}\right), \quad \boldsymbol{r}=\boldsymbol{r}_{1}-\boldsymbol{r}_{2},
$$


with corresponding momenta $\boldsymbol{P}=\boldsymbol{p}_{1}+\boldsymbol{p}_{2}$ and $\boldsymbol{p}=$ $\frac{1}{2}\left(\boldsymbol{p}_{1}-\boldsymbol{p}_{2}\right)$, the product wavefunction (8) conforms to a form separable in the center of mass and relative coordinates, each part having an anisotropic oscillator-type waveform, given by

$$
\Phi\left(\boldsymbol{R}, \boldsymbol{r} ; x_{0}\right)=N g(r) G_{a}(\boldsymbol{R}) G_{b}(\boldsymbol{r})
$$

where $N$ is the constant of normalization, and

$$
\begin{aligned}
G_{a}(\boldsymbol{R}) & =\exp \left(-\frac{1}{2} a^{2} X^{2}\right) \exp \left(-\frac{1}{2} a^{2} \lambda_{a}^{2} Y^{2}\right) \\
G_{b}(\boldsymbol{r}) & =\exp \left(-\frac{1}{2} b^{2}\left(x-x_{0}\right)^{2}\right) \exp \left(-\frac{1}{2} b^{2} \lambda_{b}^{2} y^{2}\right) .
\end{aligned}
$$

In equation (10) we have set the correlation function simply as $g(r)=\sqrt{x^{2}+y^{2}}$. This ensures $\Phi=0$ for $r=0$, so that the electrons are repulsively set separated. Moreover, such a choice for the Jastrow factor has proved to yield a comparatively lower variational upper bound to the ground state energy for the bipolaron. We should remark that the oscillator-oscillator wavefunction that we have adopted proves to be a feasible approximation [18] which, on the other hand, lends a great amount of computational simplification due to its compatibility with the quadratic barriers of the confining potential.

It should be noted that during obtaining the optimal fits to $s(q, \Phi), a, b, \lambda_{a}, \lambda_{b}$ and $x_{0}$, the variational theory is expected to set up a detailed fractional admixture of all the contributions coming from each single parameter $\left(\Omega_{x}, \Omega_{y}, U\right.$ and $\left.\alpha\right)$ characterizing the system.

The variational function $s(q, \Phi)$ of the displaced oscillator operator $(7)$ is determined from the requirement: $\partial\left\langle\Psi\left(x_{0}\right)|H| \Psi\left(x_{0}\right)\right\rangle / \partial s(q, \Phi)=0$ to yield

$$
s(q, \Phi)=\left\langle\Phi\left|2 \cos \left(\frac{1}{2} \boldsymbol{q} \cdot \boldsymbol{r}\right) \exp ( \pm \mathrm{i} \boldsymbol{q} \cdot \boldsymbol{R})\right| \Phi\right\rangle
$$

In complete form, the ground state energy (9) of the composite system of two polarons is evaluated as

$$
E_{\mathrm{g}}=E_{R}+E_{r}+\Omega_{x}^{2} E_{x}+\Omega_{y}^{2} E_{y}+E_{\mathrm{c}}+E_{\mathrm{ph}} .
$$

In the above, $E_{R}$ and $E_{r}$ stand for the kinetic terms $\left\langle\frac{1}{4} P^{2}\right\rangle$ and $\left\langle p^{2}\right\rangle$, respectively; $E_{x}$ and $E_{y}$ are the energies relevant to the confining potential; $E_{\mathrm{C}}$ is the Coulomb energy and $E_{\mathrm{ph}}$ refers to the contribution coming from the lattice. We obtain the following explicit analytic expressions for each of these terms:

$$
\begin{aligned}
& E_{R}=\frac{a^{2}}{8}\left(1+\lambda_{a}^{2}\right) \\
& E_{r}=\frac{b^{2}}{2}\left(1+\lambda_{b}^{2}\right) .
\end{aligned}
$$

Defining

$$
\begin{aligned}
& g=2 x_{0}^{2} b^{2} \\
& h=b^{2}\left[1+\lambda_{b}^{2}(1+g)\right]
\end{aligned}
$$

we calculate

$$
\begin{aligned}
E_{x} & =\left\langle\Phi\left(\boldsymbol{R}, \boldsymbol{r}, x_{0}\right)\left|X^{2}+\frac{1}{4} x^{2}\right| \Phi\left(\boldsymbol{R}, \boldsymbol{r}, x_{0}\right)\right\rangle \\
& =\frac{1}{2}\left[a^{-2}+\left(\frac{1}{2} b^{-2}+\lambda_{b}^{2} h^{-1}\right)(1+g)+\lambda_{b}^{2} g h^{-1}\right] \\
E_{y} & =\left\langle\Phi\left(\boldsymbol{R}, \boldsymbol{r}, x_{0}\right)\left|Y^{2}+\frac{1}{4} y^{2}\right| \Phi\left(\boldsymbol{R}, \boldsymbol{r}, x_{0}\right)\right\rangle \\
& =\frac{1}{2}\left[\lambda_{a}^{-2} a^{-2}+\frac{\lambda_{b}^{-2}}{2}\left(\frac{1}{2} b^{-2}+h^{-1}\right)\right] .
\end{aligned}
$$

For the Coulomb interaction we derive

$$
\begin{aligned}
E_{\mathrm{C}} & =\left\langle\Phi\left(\boldsymbol{R}, \boldsymbol{r}, x_{0}\right)\left|\frac{U}{r}\right| \Phi\left(\boldsymbol{R}, \boldsymbol{r}, x_{0}\right)\right\rangle \\
& =\frac{2 b^{3} \lambda_{b} U}{\pi h} \int_{-\infty}^{\infty} \mathrm{d} t \mathrm{e}^{-t^{2}} u_{t} \mathrm{e}^{u_{t}}\left[K_{0}\left(u_{t}\right)+K_{1}\left(u_{t}\right)\right]
\end{aligned}
$$

where $K_{0}$ and $K_{1}$ denote respectively, the modified Bessel functions of the third kind of orders zero and one, and

$$
u_{t}=\frac{1}{2} \lambda_{b}^{2}\left(t+x_{0} b\right)^{2}
$$

Letting $\phi$ denote the angle which the wavevector $\boldsymbol{q}$ makes with the $x$ axis, and setting

$$
\begin{aligned}
c_{x}^{-2} & =\frac{1}{2} a^{-2}+\frac{1}{8} b^{-2} \\
c_{y}^{-2} & =\frac{1}{2} \lambda_{a}^{-2} a^{-2}+\frac{1}{8} \lambda_{b}^{-2} b^{-2} \\
\psi & =x_{0} \cos \phi \\
\gamma^{2} & =\frac{\cos ^{2} \phi}{c_{x}^{2}}+\frac{\sin ^{2} \phi}{c_{y}^{2}} \\
A & =\frac{\lambda_{b}^{2} \cos ^{2} \phi+\lambda_{b}^{-2} \sin ^{2} \phi}{8 h} \\
B & =\frac{\lambda_{b}^{2} b^{2}}{h} \psi
\end{aligned}
$$

we write

$$
E_{\mathrm{ph}}=-\sum_{q} V_{q}^{2}[s(q, \Phi)]^{2}
$$

where

$[s(q, \Phi)]^{2}=2\left\{\left(1-A q^{2}\right)^{2}(1+\cos \psi q)+B^{2} q^{2}(1-\cos \psi q)\right.$

$$
\left.-2 B\left(1-A q^{2}\right) q \sin \psi q\right\} \exp \left(-\gamma^{2} q^{2}\right) \text {. }
$$

Introducing

$$
\xi=\frac{\psi}{2 \gamma}
$$

and defining, for notational convenience,

$$
f_{n}(\xi)=(2 \gamma)^{-(n+1)} \mathrm{e}^{-\xi^{2}} H_{n}(\xi)
$$


with $H_{n}$ denoting the nth order Hermite polynomial, and further, projecting out the $\boldsymbol{q}$-summations in equation (22), we express the lattice part of the energy in the integral form

$$
E_{\mathrm{ph}}=\frac{\alpha}{\sqrt{2 \pi}} \int_{0}^{2 \pi} \mathrm{d} \phi\left\{\frac{1}{2 \gamma}+\frac{B^{2}-2 A}{4 \gamma^{3}}+\frac{3 A^{2}}{8 \gamma^{5}}+F(\xi)\right\}
$$

where

$$
\begin{aligned}
F(\xi)=f_{0}(\xi)-2 B f_{1}(\xi)+ & \left(2 A+B^{2}\right) f_{2}(\xi) \\
& -2 A B f_{3}(\xi)+A^{2} f_{4}(\xi) .
\end{aligned}
$$

\section{Results and conclusions}

In presenting our numerical results, wherever relevant, we shall use $\Omega$ to mean $\Omega_{x}$ and/or $\Omega_{y}$. In the isotropic dot type configuration we shall simply set $\Omega=\Omega_{x}=\Omega_{y}$, and for the planar strip type configuration $\left(\Omega_{x}=0\right)$ we shall represent $\Omega_{y}$ in proportion to $\Omega$.

In regard with the strong coupling expansion to leading order in $\alpha$, i.e., $\mathcal{O}\left(\alpha^{2}\right)$, we would like to draw attention to that, if in equations $(2-3)$ the energies are scaled by $\alpha^{2}$ and lengths by $\alpha$, i.e., $E \rightarrow E \alpha^{2}$ and $L \rightarrow L \alpha$, the only modification in the Hamiltonian would be to replace the confining parameter $\Omega$ by $\Omega / \alpha^{2}$ and the Coulomb coefficient $U$ by $U / \alpha$. Thus, regardless of the value of $\Omega$, the ground state energy is seen to be proportional to the square of the coupling constant, i.e., $E_{\mathrm{g}}=-\mathcal{C} \alpha^{2}$, where the corresponding coefficient of proportionality bears a functional relation solely to $\Omega / \alpha^{2}$. Therefore, in the foregoing particular plots for which the confinement parameter $\Omega$ is expressed in the ratio $\Omega / \alpha^{2}$, one can conveniently assign $\alpha$ any arbitrary value with no loss in generality.

\subsection{Isotropic confinement}

To yield an insight into the essential role which the variational parameter $x_{0}$ in the shifted oscillator waveform (8) plays in the theory, we first provide a broad display of the variation of $E_{g}$ in the overall range of this parameter in the absence of the confining potential ( $c f$., Fig. 1a). Following the series of plots drawn for three selective values of $\eta$, we note that there shows up two asymptotic minima in the energy profiles located respectively, at $x_{0}=0$ and $x_{0} \gg 1$; the former yielding an indication of a bipolaron state, the latter representing the state of two infinitely separated non-interacting free polarons each with energy

$$
\frac{E_{\mathrm{g}}}{\alpha^{2}}=-\mathcal{C}\left(\frac{\Omega}{\alpha^{2}}\right)=-\frac{\pi}{8} \approx-0.3927
$$

We find that the particular value of $\eta$ for which the two minima have the same level is $\eta_{c}^{(2 D)}=0.158$, as given previously in references [18] and [26] in two dimensions (2D) using the same oscillator-oscillator wavefunction for the

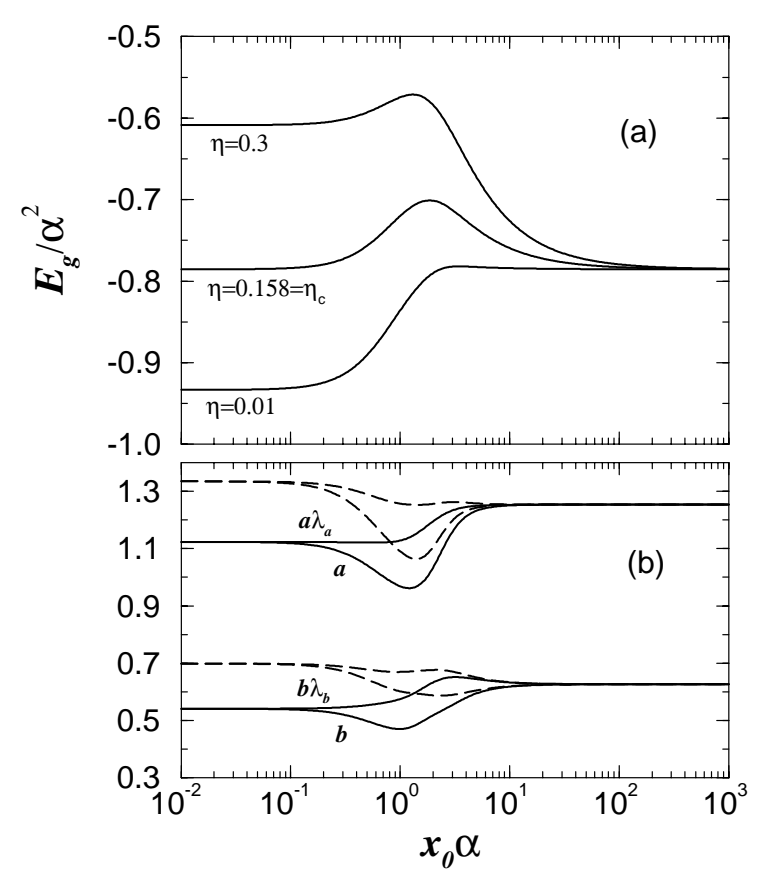

Fig. 1. (a) The ground state energy of the two-polaron complex as a function of the variational parameter $x_{0}$ for three different $\eta$ values in the absence of the confining potential. The asymptotic value on the right margin refers to the energy of two strongly coupled free polarons in two dimensions: $E_{g} / \alpha^{2}=-2(\pi / 8)$. (b) The upper set of curves represent the variational parameters $a$ and $a \lambda_{a}$ involved in the Gaussian wavefunction (11) for the center of mass coordinates. The lower set of plots describe $b$ and $b \lambda_{b}$ which are relevant to the Gaussian wavefunction (12) for the relative coordinates. The dashed (solid) curves are for $\eta=0.01(0.3)$.

electrons. Very recently, energy profiles of similar qualitative nature, separating one state from the other by a barrier, have been reported by Mukhomorov [15] in a study of the bulk-bipolaron problem in the intermediate coupling regime. Viewing the bottom curve plotted for $\eta=0.01<\eta_{c}$, we note that the minimum on the left margin lies considerably deviated below the asymptotic value $-2(\pi / 8)$ on the right margin, lending an indication in favor of an energetically stable bipolaron state. The top curve plotted for $\eta=0.3>\eta_{c}$, however, represents an example describing a metastable state of the bipolaron with most weight attributed to the state of dissociated polarons. Tuning $\eta$ to even larger values the minimum at $x_{0}=0$ loses its prominence and eventually diminishes signifying that beyond a certain strength of the Coulomb coefficient the only configuration the electrons can form is the state of separated polarons.

As complemental, we also plot the variational parameters involved in the electron-electron wavefunction (10) over the complete range of $x_{0}$ for $\eta=0.01$ and $\eta=0.3$. An immediate glance at the series of couples of curves in Figure $1 \mathrm{~b}$ reveals that in the extreme limits $x_{0}=0$ or $x_{0} \gg 1$, one essentially has a perfectly isotropic charge density of the electrons $\left(\lambda_{a}=\lambda_{b}=1\right)$ regardless of whether they form a bipolaron state or a state of dissociated polarons. In conforming from one state to the other, however, the pairs 


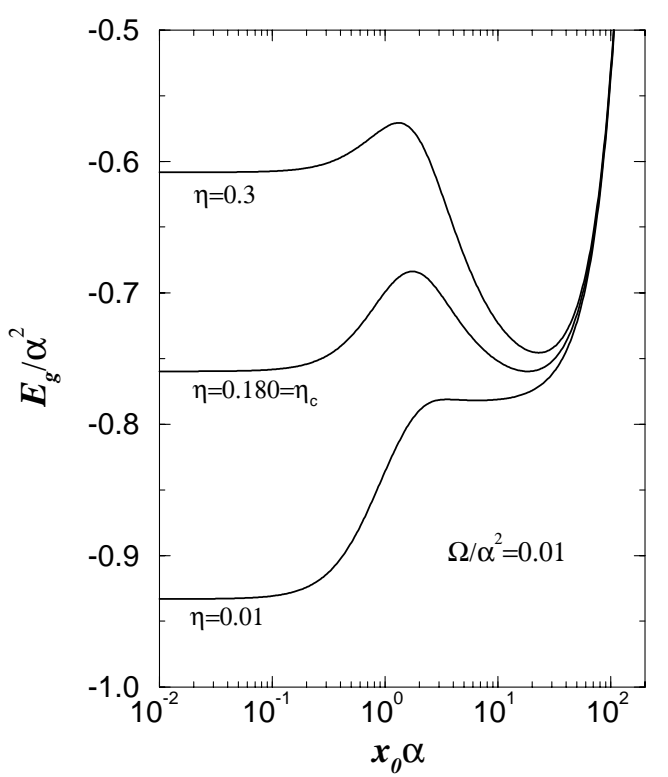

Fig. 2. The ground state energy of the two polaron complex as a function of $x_{0}$ for a succession of different $\eta$ values in the two dimensional circular quantum dot where $\Omega / \alpha^{2}=0.01$.

of curves pertaining to the $x$ and $y$ directions get splitted reflecting a strong spatial anisotropy in the transition region separating the two states of the polaron-polaron complex. We should also note that in the limit $x_{0} \gg 1$, parameters $a$ and $b$ are independent of the value of $\eta$.

Imposing the geometric confinement, one again encounters in general, two minima in the ground state energy profiles, one of which is attained asymptotically at $x_{0}=0$ reflecting the bipolaron phase, i.e., the on-center configuration of the two polarons. Referring to the curves in Figure 2 plotted for the isotropic circular geometry with $\Omega / \alpha^{2}=0.01$ (and for not too weak $\eta$ ) we observe the second minimum to take place not at infinity as in the entire space of two dimensions but, instead, at a finite value of $x_{0}$. For this ratio of $\Omega$ to $\alpha^{2}$, the critical value of $\eta$ at which the ground state energies of the on-center and offcenter configurations cross over is found to be $\eta_{c}=0.180$, a value somewhat above the two dimensional value due to the pseudo-enhancement of the effective phonon coupling under confinement. In our numerical computations we have noted that the value of $x_{0}$ increases slightly as $\eta$ is adjusted to larger values since the strengthened Coulomb interaction sets the particles apart with greater repulsion against the confining potential barriers.

In order to provide a more informative description of the effect of confinement on bipolaron formation we display the variational energy over a series of plots for different $\Omega$ with $\eta=0.2$ ( $c f .$, Fig. 3). Following the succession of curves from bottom to top, one traces the transition from a configuration of two separated polarons to that of a stable bipolaron. One readily observes that, starting from the entire two dimensional configuration and turning on the confinement, the polarons in the off-centered configuration get inevitably closer as the degree of confinement is increased. In the meantime, the corresponding minimum of energy increases in value and beyond a certain strength

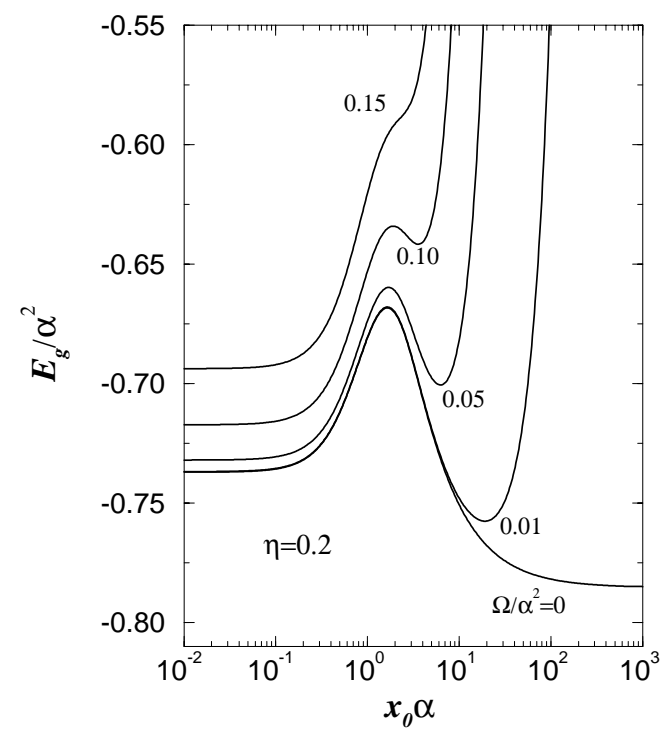

Fig. 3. The ground state energy of the two polaron complex as a function of $x_{0}$ in the two dimensional circular quantum dot where $\eta=0.2$. The succession of curves from bottom to top are for $\Omega / \alpha^{2}=0,0.01,0.05,0.1$ and 0.15 .

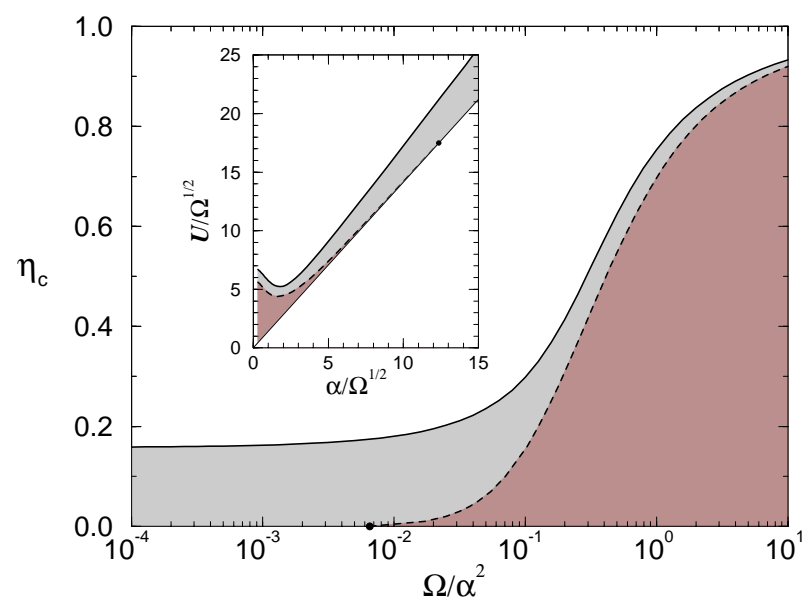

Fig. 4. The phase diagrams illustrating the domain of stability of the bipolaron phase in terms of the critical ratio $\eta$ and the Coulomb coefficient $U$ as a function of the confining parameter $\Omega$ in a circular dot. In either figure, the solid curve is the phase boundary above (below) which the off- (on-) center configuration occurs more favorably. In the dark gray region bounded from above by the dashed line the two polaron complex is found to take solely the bipolaron configuration.

of the potential barriers $\left(\Omega / \alpha^{2}>0.025\right)$ crosses over the minimum relevant to the on-center configuration representing a bipolaron state. Thus, in the overall, the effect of confinement is seen to favor the formation of bipolarons and for dots of small size (as exemplified by the topmost curve), the bipolaron state may even turn out to be the only and unique ground state of the two-polaron assembly.

Finally, pertaining to the planar circular dot geometry we display the phase diagrams for bipolaron formation in Figure 4, where we plot the critical Coulomb strength as a function of $\Omega$. In the main figure and in the inset respectively, we present the critical parameter $\eta_{c}$ and the 
alternative related Coulomb amplitude $U$ against $\Omega / \alpha^{2}$ plotted universally for any given value of $\alpha$. The solid curve which starts at value $\eta_{c}^{(2 D)}=0.158$ on the left margin is the main boundary $\left(\eta=\eta_{c}\right)$ along which the two minima corresponding to the off-center (polaron-polaron: $\mathrm{PP}$ ) and the on-center (bipolaron: BP) configurations have the same level of energy. Above and below this boundary (empty and shaded regions) one respectively has either the $\mathrm{PP}$ or the BP phase to take place more favorably, dominating the occurrence of one over the other. In the region below the dashed boundary (shaded dark gray) the variational calculation yields $x_{0}=0$ as the only and unique fit to this parameter. In this region the polarons are thus realized to conform totally to the on-center bipolaronic configuration. The same qualitative trait can be retrieved from the inset picturing the domain of stability of the bipolaron phase in the $U-\alpha$ plane. The essential aspect which one notes from either representation of the phase diagram in Figure 4 is that, contrary to most outcomes of the recent literature [24-27] (pertaining to three dimensional dots), the confinement effects act in favor of bipolaronic stability and, even for not too strong $\alpha$, bipolarons can form in small-radius dots.

\subsection{Anisotropic confinement}

Having applied our variational model to two dimensional dots of disk type, we now would like to extend our considerations to the study of bipolarons confined within dots of anisotropic nature, where we take $\Omega_{y} \geq \Omega_{x}$. As an extreme and interesting case, we would also like to consider planar wires. Setting $\Omega_{x}=0$ and adjusting $\Omega_{y}$ to nonzero arbitrary values one achieves a description of a strip-type geometry, and even a narrow quasi-one dimensional wire-like configuration. In order to provide a broad and comparative layout of the basic aspects of bipolaron formation in a general sense encompassing the circular geometry on one side and the planar wire on the other, and to show explicitly the distinguishing characteristics of the two geometries, we introduce an anisotropy parameter $\mu$ ranging within zero and unity, given by

$$
\begin{aligned}
& \Omega_{x}=(1-\mu) \Omega \\
& \Omega_{y}=(1+\mu) \Omega .
\end{aligned}
$$

Such a representation, besides allowing us to keep an explicit trace of the effect of confinement, leaves the subband energy invariant over the complete range of $\mu$.

In Figure 5 we plot the variational ground state energies pertaining to the bipolaron and polaron-polaron phases as a function of $\mu$ for two different degrees of confinement: $\Omega / \alpha^{2}=0.1$ and 0.2 . The overall implication led by both pairs of curves plotted for $\eta=0.2$ is that the effect of anisotropy is to inhibit bipolaron formation. Following the set of plots from left to right, we observe that the BP phase which is seen to have considerably deeper binding than the PP phase for $\mu=0$ turns out to become unfavorable as the degree of anisotropy is increased. This peculiar feature is even more striking for higher degrees

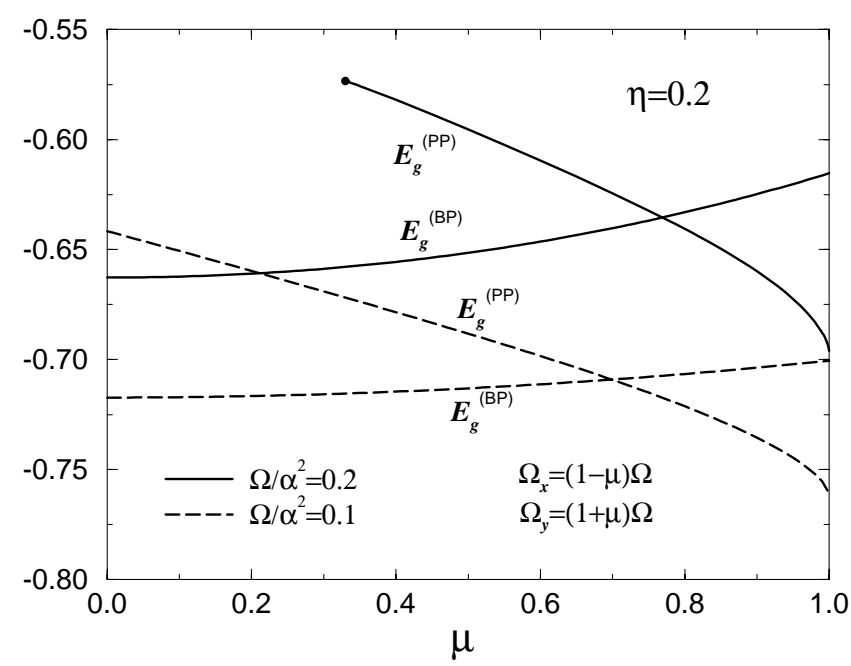

Fig. 5. The variational ground state energies (scaled by $\alpha^{2}$ ) of the two-polaron complex in the bipolaron (BP) and polaron-polaron (PP) phases as a function of $\mu$, the degree of anisotropy. The dashed (solid) curves are for $\eta=0.2$ and $\Omega / \alpha^{2}=0.1(0.2)$.

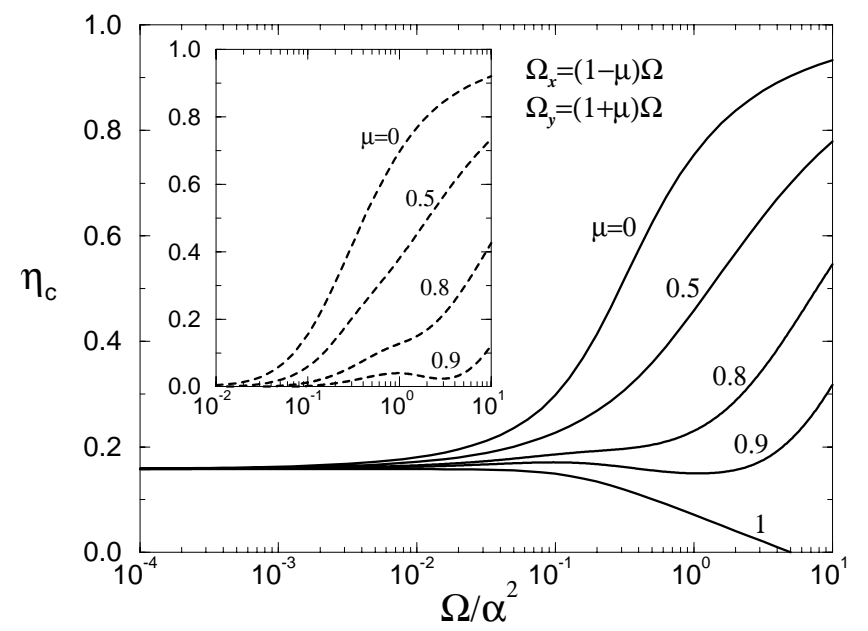

Fig. 6. The phase boundaries separating the $\mathrm{BP}$ and $\mathrm{PP}$ phases in the space $\eta$ versus $\Omega$ for different degrees of anisotropy: $(\mu=0,0.5,0.8,0.9,1)$. The value to which all the plots merge on the left margin is $\eta_{c}^{(2 D)}=0.158$. The dashed curves in the inset are the boundaries below which the two polaron complex is found to take solely the bipolaron configuration.

of confinement. For $\Omega / \alpha^{2}=0.2$, for instance, one has the $\mathrm{BP}$ phase to show up as the only and unique configuration of the two-polaron complex for $0 \leq \mu<0.33$. On the other extreme $(\mu \rightarrow 1)$, however, it is dominantly the PP phase to exist. One therefore observes that, in tuning $\mu$ from small values to unity, the bipolaron state breaks up and dissociates into two separate polarons. An alternative description of this salient feature is given in Figure 6 where we picture a sequence of phase boundary curves, $\eta_{c}$ versus $\Omega$, for different degrees of anisotropy. The solid 
curves in the main figure give the boundary separating the two domains where either the bipolaron state is energetically more stable and the off-center two-polaron configuration is metastable, or vice versa, depending respectively, on whether $\eta<\eta_{c}$ or $\eta>\eta_{c}$. In the inset, we plot the relevant boundaries below which the bipolaron state is the only and unique configuration which the complex can take. The topmost curves in both the main figure and the inset, plotted for $\mu=0$, constitute a replica of Figure 4 and represents the perfectly isotropic disk-type confinement. The bottom curve relevant to $\mu=1$ reflects an entirely distinguishing case of an open ended planar strip the width of which is determined by the parameter $\Omega$. The intermediate curves plotted for $\mu=0.5,0.8$ and 0.9 give the corresponding phase boundaries for the BP-PP transition pertaining to the anisotropic elliptic confinement geometry. A careful glance at the sequence of solid curves drawn for each of the above geometric configurations reveals that the effect of confinement is either to enlarge the bipolaron stability region or to act in opposition to yield dissociated polarons, depending respectively, on whether the anisotropy parameter takes on values close to zero or unity. In the peculiar case of an open ended planar strip $\left(\Omega_{y}>\Omega_{x}=0, \mu=1\right)$ the phase profile displays a contrasting behavior deviated strongly from the general trend portrayed by all the other plots drawn for $\mu<1$. Instead of having the stability region to be broadened or at least conserved, we observe that in the planar strip configuration the critical parameter $\eta_{c}$ gets considerably lowered with increasing $\Omega$, and eventually diminishes in the limit of a thin planar wire. The underlying reason which sets the form of $\eta_{c}$ plotted for $\mu=1$ apart from those for $\mu<1$ is that in the circular or elliptic type confinements the electrons do not have any free spatial direction to expand. However, in the open ended strip the degree of localization of the charge density of the electrons decreases, which in turn, leads to a weaker effective coupling of the electron pair to the phonon field. Consequently, it becomes less likely for the phonon-mediated attraction to withstand against the repulsive Coulomb interaction and this makes the bipolaron state easier to split and dissociate into two individual single polaron states. We would also like to draw attention to that, while in closed confinements $(\mu<1)$ one encounters regions relevant solely to the bipolaron phase, in the strip or wire geometry one does not meet any such region ( $c f$., the inset of Fig. 6).

In this article we have considered the problem of formation of two dimensional bipolarons confined within a two-axis elliptic potential well of quadratic barriers. The bipolaron model adopted here involves the polaronpolaron separation introduced as a free parameter which one determines variationally together with the interrelated and competitive roles of all the parameters which characterize the system. Thus, rather than founding the stability criterion on a comparison of the energy of the presumed bipolaron state with that of two noninteracting polarons in similar individual dots of the same size and of identical material parameters, we have chosen to treat the confined electrons to constitute either a bound bipolaronic state or go into a state of two individual polarons in essentially the same dot. Within the framework of this diverting approach applied to circular dots, we find that the fundamental effect of the confining potential is to enlarge the region of bipolaron formation and to sustain the bipolaron phase even for small radii in contrast with most results led by the preceding literature [24-27] pertaining to spherical dots. Extending our calculations to anisotropic geometric configurations we further observe that the effect of anisotropy is to inhibit bipolaron formation, and that this contravening aspect to become even more prominent in a confining potential with steeper barriers.

\section{References}

1. S.I. Pekar, Research on Electron Theory in Crystals [US AEC, Washington DC, 1963].

2. V.L. Vinetskii, M.S. Gitterman, Zh. Eksp. Teor. Fiz. 33, 730 (1957) [Sov. Phys. JETP 6, 560 (1958)].

3. Y. Takada, Phys. Rev. B 26, 1223 (1982).

4. H. Hiramoto, Y. Toyozawa, J. Phys. Soc. Jpn 54, 245 (1985).

5. J. Adamowski, Phys. Rev. B 39, 3649 (1989).

6. T.K. Mitra, Phys. Lett. A 142, 398 (1989).

7. V. Cataudella, G. Iadonisi, D. Ninno, Phys. Scr. T 39, 71 (1991).

8. F. Bassani, M. Geddo, G. Iadonisi, D. Ninno, Phys. Rev. B 43, 5296 (1991)

9. J. Adamowski, S. Bednarek, J. Phys. Cond. Matt. 4, 2845 (1992).

10. A. Chatterjee, S. Sil, Int. J. Mod. Phys. B 7, 4763 (1993).

11. P. Vansant, M.A. Smondyrev, F.M. Peeters, J.T. Devreese, J. Phys. A 27, 7925 (1994).

12. C. Qinghu, W. Kelin, W. Shaolong, Phys. Rev. B 50, 164 (1994).

13. S. Sahoo, J. Phys. Cond. Matt. 7, 4457 (1995).

14. M.A. Smondyrev, J.T. Devreese, F.M. Peeters, Phys. Rev. B 51, 15008 (1995).

15. V.K. Mukhomorov, J. Phys. Cond. Matt. 13, 3633 (2001).

16. G. Verbist, F.M. Peeters, J.T. Devreese, Phys. Rev. B 43, 2712 (1991)

17. S. Sil, A.K. Giri, A. Chatterjee, Phys. Rev. B 43, 12642 (1991).

18. G. Verbist, M.A. Smondyrev, F.M. Peeters, J.T. Devreese, Phys. Rev. B 45, 5262 (1992).

19. F. Luczak, F. Brosens, J.T. Devreese, Phys. Rev. B 52, 12743 (1995).

20. R.T. Senger, A. Erçelebi, Phys. Rev. B 60, 10070 (1999).

21. R.T. Senger, A. Erçelebi, Phys. Rev. B 61, 6063 (2000).

22. E.P. Pokatilov, V.M. Fomin, J.T. Devreese, S.N. Balaban, S.N. Klimin, Phys. Rev. B 61, 2721 (2000).

23. Y. Wan, G. Ortiz, P. Phillips, Phys. Rev. B 55, 5313 (1997).

24. S. Mukhopadhyay, A. Chatterjee, J. Phys. Cond. Matt. 8, 4017 (1996).

25. E.P. Pokatilov, V.M. Fomin, J.T. Devreese, S.N. Balaban, S.N. Klimin, J. Phys. Cond. Matt. 11, 9033 (1999).

26. R.T. Senger, A. Erçelebi, Eur. Phys. J. B 16, 439 (2000).

27. A. Chatterjee, S. Mukhopadhyay, Acta Phys. Polonica B 32, 473 (2001). 
28. R.T. Senger, A. Erçelebi, unpublished.

29. R. Micnas, J. Ranninger, S. Robaszkiewicz, Rev. Mod. Phys. 62, 113 (1990).

30. D. Emin, Phys. Rev. Lett. 62, 1544 (1989).

31. J.T. Devreese, in Encylopedia of Applied Physics, edited by G.L. Trigg (VCH, Weinheim, 1996), Vol. 14, pp. 383-413.

32. A.S. Alexandrov, N.F. Mott, Rep. Prog. Phys. 57, 1197 (1994).
33. J. Duan, G.G. Bishop, E.S. Gillman, G. Chern, S.A. Safron, J.G. Skofronick, Vac. Sci. Technol. A 10, 1999 (1992).

34. S.A. Safron, J. Duan, G.G. Bishop, E.S. Gillman, J.G. Skofronick, J. Phys. Chem. 97, 1749 (1993).

35. R.R. Hudgins, P. Durourd, J.M. Tenenbaum, M.F. Jarrold, Phys. Rev. Lett. 78, 4213 (1997). 